\section{THE ANALYTICAL LIMITS: HADF (High Angle Dark Field Imaging)}

Michael Kersker, JEOL USA

High Angle Dark Field Imaging, or $\mathrm{Z}$ contrast imaging, is an IMAGING method. It takes advantage of the useful fact that if one uses the high angle scattering intensities and eliminates the elastic scattered (diffracted) beams from the image (by using a Howie type angular dark field detector), the remaining image will be characterized by, if the probe used is on the order of the atomic dimensions, intensity modulations that reveal atom positions and relative atomic number. In simple terms, the image will display Z-contrast at the atomic level and can differentiate columns of heavy atoms from columns of lighter ones. Due to channeling effects, the effective beam is localized along the atomic columns. There is no loss of resolution with increasing crystal thickness, a counterintuitive result given that increasing thickness would seem to mean increased probability of scattering away from the column. The images thus formed are reasonably intuitive. There is no contrast reversal when specimen thickness is increased or decreased. Blobs and spots are in positions where atoms should be provided the probe sizes employed are small enough to resolve the effective atom spacings. In short, Z-contrast imaging is a truly high resolution method for imaging crystals AND single supported atoms provided a field emission STEM with a suitable small probe is used. (See Pennycook et al, 1995, for an excellent review).

The image on the cover of this month's Microscopy Today is an excellent example of the resolution achievable with a small probe STEM instrument. The image shows a grain boundary in $\mathrm{Si}$. The two crystals have identical orientations but are rotated 39 degrees with respect to one another. The spacings between spots in each dumbbell are 0.136 $\mathrm{nm}$. It is clear from the image which atoms are associated with which crystal and consequently the boundary structure is clearly resolved. Other examples of boundary structures analysis can be found in McGibbon et al, 1996.

We have recently looked at $\mathrm{Sr} T i 03$ to see if it is possible to resolve in $\mathrm{HADF}$, the $\mathrm{Sr}$ and $\mathrm{Ti}$ using a conventional FEGTEM with a scanning attachment. The crystals were prepared by Nigel Browning, University of Illinois at Chicago, and were analyzed in a JEM 2010F with Schottky thermal field emission source. Analyzed crystal thickness was on the order of $30-40 \mathrm{~nm}$. Samples were cleaned in a small plasma cleaner prior to insertion in the microscope. The polepiece constants for the $2010 \mathrm{~F}$ were $\mathrm{Cs}=1.0 \mathrm{~mm}$ and $\mathrm{Cc}=1.4 \mathrm{~mm}$ giving a theoretical probe size of $0.15 \mathrm{~nm}$ for this lens configuration. High Angle Dark Field images were captured using an angular scintillator PMT coupled dark field detector (approximate angular range 40 mrad - $120 \mathrm{mrad}$ ) and a GATAN Digiscan digitizing interfaces Imaging was accomplished by using the finest probe we could achieve with the $2010 \mathrm{~F}$. The probe thus formed, though not accurately measured, appeared to be slightly less than $0.2 \mathrm{~nm}$ Probe current was on the order of $20 \mathrm{pA}$.

Results are shown in figures 1 and 2. Figure 1 shows the raw data. The bright spots in this image are the $\mathrm{Sr}$ atoms They are $0.39 \mathrm{~nm}$ apart. The Ti atoms sit at the intersection o 8 the diagonals of these $\mathrm{Sr}$ squares. The $\mathrm{Ti}$ signal is wea compared to the $\mathrm{Sr}$ signal. Figure 2 is an image enhanced bo Fourier filtering and intensity coloring. The $\mathrm{Ti}$ atoms can be clearly seen in the diagonal positions. $\mathrm{Sr}$ to $\mathrm{Ti}$ spacing is 0.2 䨌 $\mathrm{nm}$.

Though the images show excellent resolution and reveag something of the atomic structure, an additional benefit of such high resolution would be the measurement of chemicas profiles with single column resolution. This was demonstrated by Browning et al. (Browning et al, 1993) using PEEL क्षे measurements. Beam currents in the $2010 \mathrm{~F}$ are adequate fo this measurement though no profiling data presently exists Figure 3 shows a PEELS spectrum obtained by using a nomi nal $0.2 \mathrm{~nm}$ probe and an 8 second acquisition. Since the beam has to dwell for a significant time at one point on the sample,

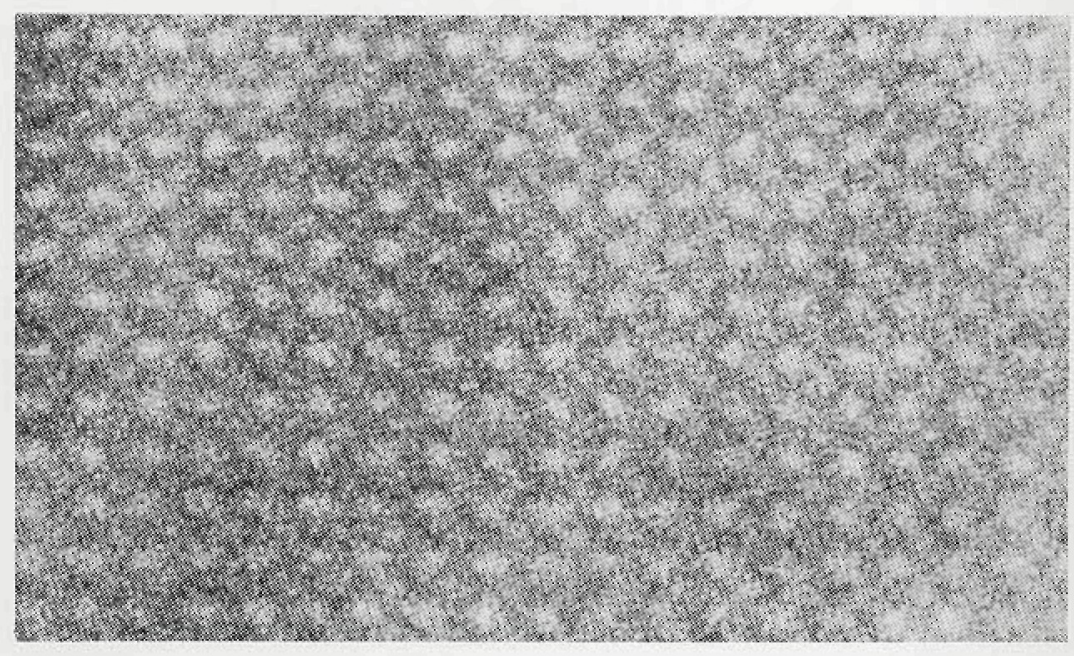

Figure 1: Z Contrast image of [100] SrTi03, unprocessed image.

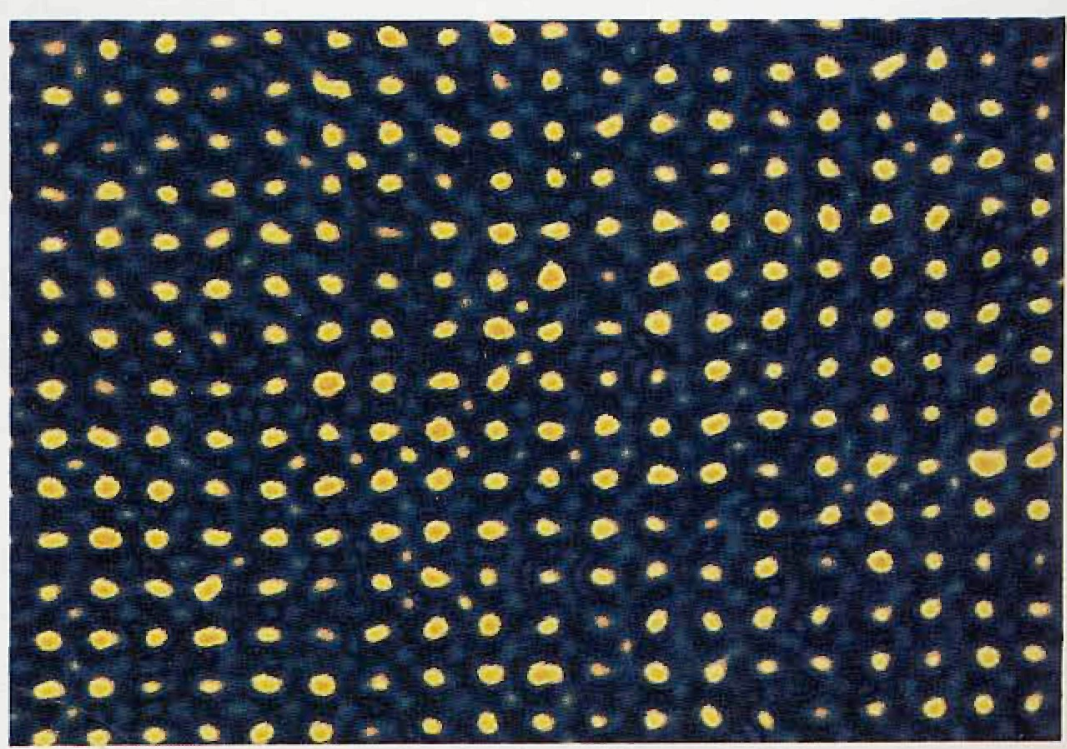

Figure 2: Filtered and enhanced image of Figure 1. 
the microscope stability must be exceptional to achieve single column resolution. Clearly, stage stability is very important since typical PEELS acquisitions can take upward of 10 seconds per spectrum. The microscope used in this experiment employs a new fine piezo adjusted stage that can compensate for stage drift in increments as small as $0.01 \mathrm{~nm}$, thus overcoming one of the most difficult barriers for accurate analysis ... knowing where the $0.2 \mathrm{~nm}$ probe is placed with an accuracy of better than $0.1 \mathrm{~nm}$ for the sensible time it takes to make a measurement at that point.

$\mathrm{Z}$ Contrast Imaging is an exciting new field and is now one not necessarily limited, as in the past, to those researchers with dedicated STEM instruments. We have demonstrated high resolution $\mathrm{Z}$ contrast imaging in a conventional FEGTEM with a commercially available scanning unit. Watch these pages in the future for more information on, and applications for, this very high resolution method of image and chemical analysis. Browing, N.D., Chisholm, M.F. and Pennycook, S.J. (1993) Atomic Resolution Chemical Analysis Using a Scanning Transmission Electron Microscope, Nature, 366, 143-146.

McGibbon, M.M., Browning, N.D., McGibbon, A.J. and Pennycook, S.J. (1996), The Atomic Structure of Asymmetric [001] Tilt Boundaries in SrTi03, Philosophical Magazine A, Vol. 73, 625-641.

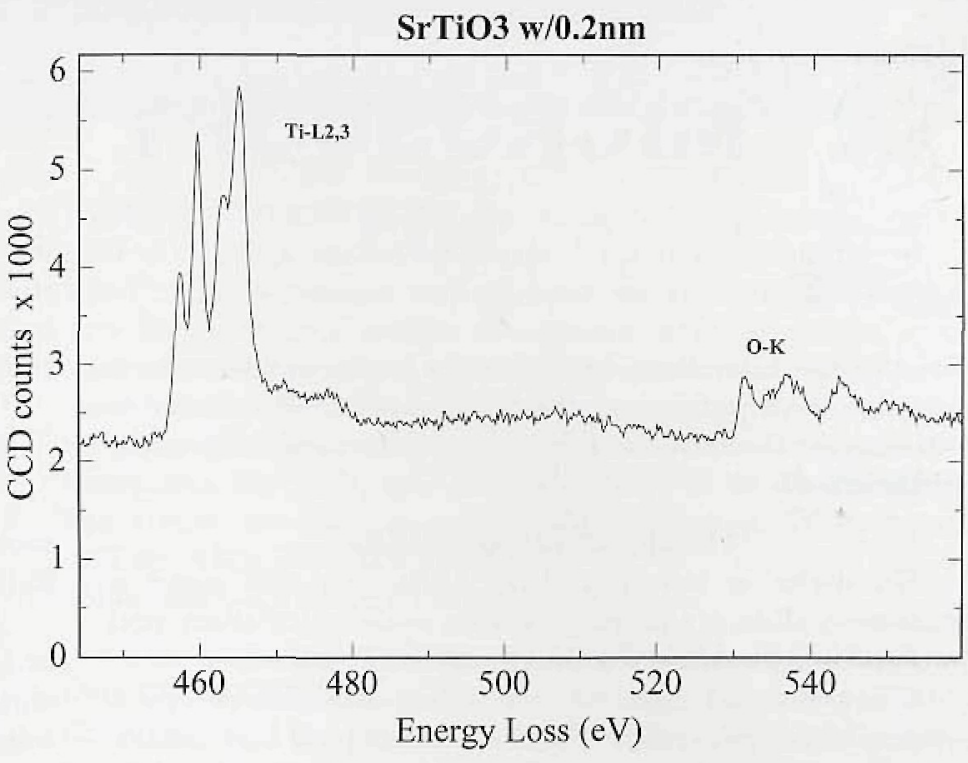

Figure 3: PEELS spectrum from SrTi03 using a $0.2 \mathrm{~nm}$ probe. Pennycook, S.J., Jesson, D.E., Chisholm, M.F., Browing, N.D., McGibbon, A.J. and McGibbon (1995), M.M., ZContrast Imaging in the Scanning Transmission Electron Microscope, Journal of the Microscopy Society of America, Vol 1, Number 6, 231-251.

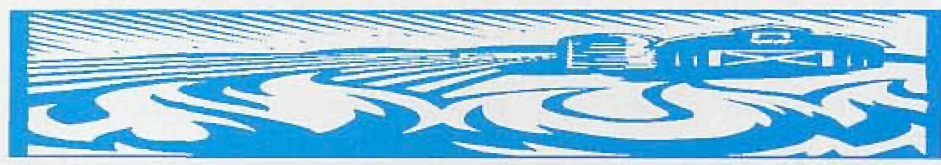

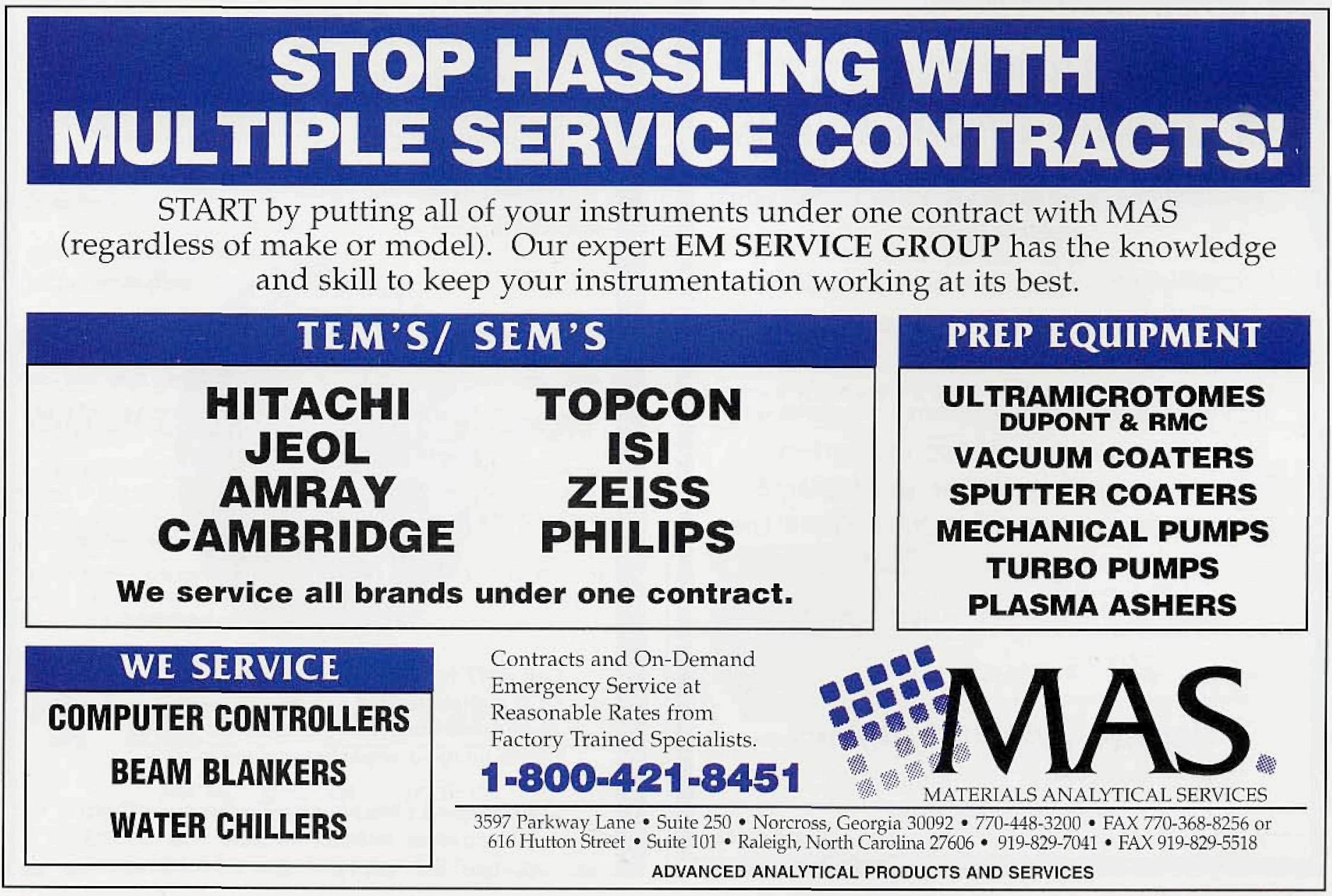

